\title{
洋葱碳的制备与应用研究进展
}

\author{
郑艳涁 ${ }^{1,2}$, 姜志刚 ${ }^{1}$, 朱品文 ${ }^{1}$ \\ (1. 吉林大学 超硬材料国家重点实验室, 长春 130012; 2. 长春中医药大学, 长春 130117)
}

摘 要: 洋苟碳独特的结构, 使其具有优异的物理化学性能。本文首先介绍了洋䓤碳的分类和结构, 对几种传统的 制备方法(包括电弧放电法、等离子体、电子束辐射、化学气相沉积、纳米金刚石真空退火、热解法)的优缺点进行 归纳、总结。其次, 介绍了近年来发展起来的制备方法。随后，对近年来洋苾碳在锂离子二次电池负极、染料敏化 太阳能电池对电极、电化学储氢电极、超级电容器电极、摩擦和磨损、催化领域的应用做一概述。最后, 指出了目 前洋苾碳在制备和应用方面的不足，对今后的研究做了展望。

关 键 词: 洋苾碳; 制备方法; 应用; 研究进展

中图分类号: TQ127 文献标识码: A

\section{Development on the Preparation and Application of Onion-like Carbon}

\author{
ZHENG Yan-Bin ${ }^{1,2}$, JIANG Zhi-Gang ${ }^{1}$, ZHU Pin-Wen ${ }^{1}$
}

(1. State Key Laboratory of Superhard Materials, Jilin University, Changchun 130012, China; 2. Changchun University of Chinese Medicine, Changchun 130117, China)

\begin{abstract}
Due to unique structure, onion-like carbon has excellent physical and chemical properties that brodens the application in carbon and carbon-based composite materials. The classification and the structure of onion-like carbon were firstly introduced. Its advantages and disadvantages of traditional preparation methods, including arc discharge, plasma, electron-beam radiation, chemical vapor deposition, nanodiamond annealing in vacuum, thermolysis, were summarized. Then, some new synthetic methods developed in recent years were also presented. Subsequently, the application of onion-like carbon on anode materials of lithium ion secondary battery, counter electrode materials of dye-sensitized solar cell, electrodes materials of electrochemical hydrogen storage and super capacitor, friction and wear, and catalyst fields was summarized. Finally, deficiencies of preparation and application on onion-like carbon were pointed out and future research were put forward.
\end{abstract}

Key words: onion-like carbon; preparation methods; application; research progress

洋葱碳(Onion-like Carbon, 简称 OLC), 又称为 碳纳米洋苾(Carbon Nano-onions, 简称 CNOs), 其 尺寸大约在 3 50 $\mathrm{nm}^{[1]}$, 是继 $\mathrm{C}_{60}$ 、碳纳米管(Carbon Nano-tubes, 简称 CNTs)之后, 富勒烯家族的新成 员。洋苟碳按其结构一般可分为两类: 中空结构的
洋苟碳和核壳结构的洋䓤碳。理想的具有中空结构 的洋葱碳是由同心的球形石墨壳层嵌套而成, 如图 (a)所示, 其最内层是一个 $\mathrm{C}_{60}$ 分子(由 60 个碳原子 组成, 其直径约为 $0.7 \mathrm{~nm}$ )。由内往外, 中空结构的洋 苾碳每一石墨壳层所含碳原子数为 $60 n^{2}$ ( $n$ 代表层数)

收稿日期：2014-12-16; 收到修改稿日期：2015-03-24

基金项目：国家自然科学基金(51172091); 教育部新世纪优秀人才支持计划(NCET-12-0240); 国家基础科学人才培养基金 (J1103202); 吉林省科技发展计划项目(20130101023JC)

National Natural Science Foundation of China(51172091); Program for New Century Excellent Talents in University of Ministry of Education of China(NCET-12-0240); National Found for Fostering Talents of Basic Science(J1103202); Jilin Province Science and Technology Development Program, China(20130101023JC)

作者简介：郑艳彬(1983-), 男, 讲师，博士研究生. E-mail: zhengyb14@mails.jlu.edu.cn

通讯作者：朱品文, 教授. E-mail: zhupw@jlu.edu.cn; 姜志刚, 教授. E-mail: jiangzg@jlu.edu.cn 


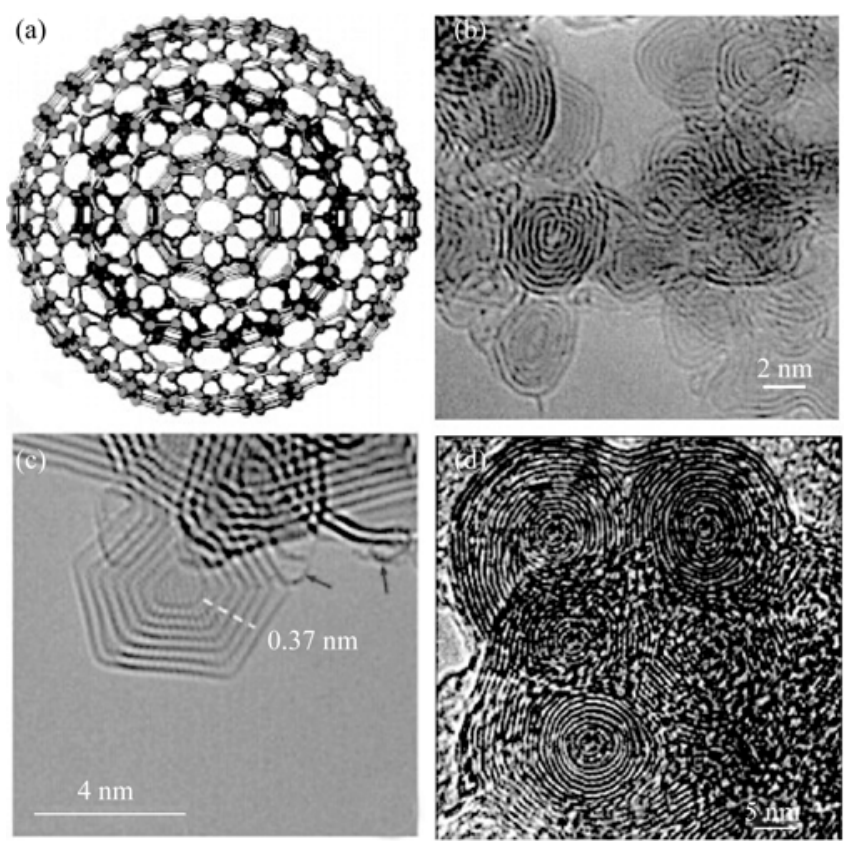

图 1 (a)理想的中空 OLC 的结构模型 ${ }^{[7]}$, (b)准球形结构的 $\mathrm{OLC}^{[8]}$, (c)多面体结构的 OLC ${ }^{[9]}$ 和(d)多核结构的 OLC ${ }^{[10]}$

Fig. 1 (a) Structure model of ideal hollow OLC ${ }^{[7]}$ with (b) quasi-spherical structure ${ }^{[8]}$, (c) polyhedral structure ${ }^{[9]}$ and (d) multi-core structure ${ }^{[10]}$

相邻层的间距约为 $0.34 \mathrm{~nm}$ 。核壳结构洋葱碳的核可以 是过渡金属粒子 ${ }^{[2]}$ 、过渡金属氧化物 ${ }^{[3-5]}$ 和纳米金刚石 颗粒 ${ }^{[6]}$ 等, 其壳由球形的石墨层嵌套而成。洋葱碳通常 并不是严格的同心石墨壳层嵌套组成的球形结构, 大 部分呈现出准球形结构或多面体结构, 如图 1(b)与(c) 所示。在一定条件下, OLC 的外层石墨层会连接在一起, 形成具有多核结构的洋忽碳, 如图 1(d)所示。

洋苾碳和纳米石墨微粒是两个不同的概念。纳米 石墨微粒包括中空结构和核壳结构的洋葱碳, 也包括 那些壳层排列并不规则, 最内层常常存在尺寸不等空 隙的微粒。中空的, 壳层呈不规则形状的纳米石墨微粒, 其尺寸一般大于中空的洋䓤碳, 结构和稳定性更趋近

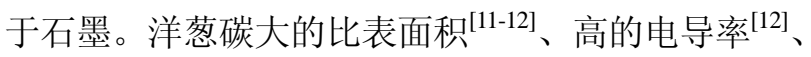

高的热稳定性以及封闭稳定的结构使其具有较大的应 用潜力, 在催化 ${ }^{[13]}$ 、摩擦 ${ }^{[14-16]}$ 、锂离子二次电池 ${ }^{[17-18]}$ 、 太阳能电池 ${ }^{[19]}$ 、电化学储氢 ${ }^{[20-21]}$ 、超级电容器 ${ }^{[22-24]}$ 和 电磁屏蔽 ${ }^{[25]}$ 等领域都显示出良好的应用前景。

\section{1 洋葱碳的制备方法}

洋怱碳的制备已有 20 多年历史, 制备方法较多, 可分为物理方法和化学方法两类。物理方法主要有

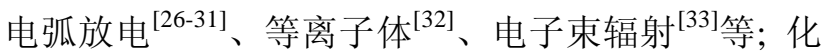
学方法主要有化学气相沉积法 ${ }^{[34-37]}$ 、纳米金刚石真 空热处理 ${ }^{[38-40]}$ 、热解法 ${ }^{[41]}$ 等, 上述制备方法的优缺 点, 如表 1 所示。

\section{表 1 OLC 的主要制备方法优缺点比较}

Table 1 Advantages and disadvantages of OLC's main preparation methods

\begin{tabular}{|c|c|c|}
\hline Preparation methods & Advantages & Disadvantages \\
\hline Arc discharge & $\begin{array}{l}\text { High crystallization, low defect density } \\
\text { carbon onions prepared in bulk quantities } \\
\text { using an arc in water }\end{array}$ & $\begin{array}{l}\text { Containing a large amount of carbonaceous impuri- } \\
\text { ties such as amorphous carbon, CNTs, CNT-like } \\
\text { structures, graphitic debris, and metallic impurities }\end{array}$ \\
\hline Plasma & Low cost and prepared in bulk quantities & $\begin{array}{l}\text { Containing amorphous carbon and metallic impu- } \\
\text { rities }\end{array}$ \\
\hline Electron-beam radiation & $\begin{array}{l}\text { In-situ observations achieved helpful to } \\
\text { study the growth mechanism of OLC }\end{array}$ & High cost and low yield \\
\hline Chemical vapor deposition & $\begin{array}{l}\text { Simple, cheap, easy to implement and to } \\
\text { realize mass production }\end{array}$ & $\begin{array}{l}\text { Purification to remove amorphous carbon, CNTs, } \\
\text { graphite, catalyst support, etc }\end{array}$ \\
\hline $\begin{array}{l}\text { Nanodiamond annealing in } \\
\text { vacuum }\end{array}$ & $\begin{array}{l}\text { Narrow-sized distribution and produced in } \\
\text { massive amount }\end{array}$ & $\begin{array}{l}\text { Requiring nanodiamond precursor and } \\
\text { high-temperature vacuum oven }\end{array}$ \\
\hline Thermolysis & $\begin{array}{l}\text { Massive amount of carbon onions produced } \\
\text { at low cost by using simple device }\end{array}$ & Larger size CNOs and/or purification requirement \\
\hline
\end{tabular}


激光辐照法也是一种可以快速宏量制备洋葱碳的 方法, 而且它使用的碳源种类丰富, 包括非晶碳化硅 ${ }^{[42] 、 \text { 炭黑 }}{ }^{[43]}$ 、石墨 ${ }^{[44]}$ 、丙酩 ${ }^{[45]}$ 、乙烯 ${ }^{[46]}$ 等。最近, Xiao 等 ${ }^{[47]}$ 发现, 用激光辐照纳米金刚石也可以制备出洋葱 碳, 并且可以实现纳米金刚石一洋䓤碳的可逆转换。纳 米金刚石向洋葱碳的转变是由于激光诱导的高温造成 的; 洋苟碳向纳米金刚石的转变是由激光诱导的高温 以及高压两方面共同作用的结果。OLC 的制备方法还 有碳离子注入法 ${ }^{[48]}$ 和球磨法 ${ }^{[49]}$ 等。

但上述制备方法都涉及到以下几个问题：高 能消耗; 工艺复杂、可控性低; 伴有杂质相和产量 低。近年来, 相继出了一些新的 OLC 制备方法。 Han 等 ${ }^{[50}$ 通过 $\mathrm{CuCl}_{2} \cdot 2 \mathrm{H}_{2} \mathrm{O}$ 和 $\mathrm{CaC}_{2}$ 在 $600^{\circ} \mathrm{C}$ 反应 生成了高纯度、粒度分布均匀的(大约 $30 \mathrm{~nm}$ )OLC。 Ghosh 等 ${ }^{[51]}$ 通过先碳化木材废料, 再后经浓硝酸 处理, 制备出水溶性的 OLC。Choucair 等 ${ }^{[52]}$ 通过燃 烧荟制备出克量级的 OLC, 制备过程不需催化剂, 并且产物无任何杂质。

\section{2 洋葱碳的应用}

\subsection{OLC 在锂离子二次电池负极材料方面的 应用}

石墨类碳材料作为锂离子电池的负极材料, 目 前已实现商业化。但是石墨的理论比容量低 (372 mAh/g), 限制了锂离子电池比能量的进一步提 高。由于金属氧化物具有高的理论比容量(如表 2), 一些环境友好、制造成本低的金属氧化物已引起研 究者的兴趣。在充放电循环过程中负极材料由于体

表 2 不同金属氧化物的理论比容量

Table 2 Theoretical capacities of various metal oxides

\begin{tabular}{ccc}
\hline Metal oxides & Theoretical capacity $/\left(\mathrm{mAh} \cdot \mathrm{g}^{-1}\right)$ & Ref \\
\hline $\mathrm{SnO}_{2}$ & 782 & {$[54]$} \\
$\mathrm{CuO}$ & 674 & {$[4]$} \\
$\mathrm{CdO}$ & 1046 & {$[55]$} \\
$\mathrm{Cr}_{2} \mathrm{O}_{3}$ & 1058 & {$[56]$} \\
$\mathrm{MoO}_{2}$ & 838 & {$[57]$} \\
$\mathrm{NiO}$ & 718 & {$[3]$} \\
$\mathrm{MnO}$ & 755 & {$[58]$} \\
$\mathrm{MnO}_{2}$ & 1233 & {$[59]$} \\
$\mathrm{Mn}_{2} \mathrm{O}_{3}$ & 1019 & {$[60]$} \\
$\mathrm{Fe}_{2} \mathrm{O}_{3}$ & 1007 & {$[60]$} \\
$\mathrm{Fe}_{3} \mathrm{O}_{4}$ & 926 & {$[61]$} \\
$\mathrm{CoO}$ & 715 & {$[62]$} \\
$\mathrm{Co}_{3} \mathrm{O}_{4}$ & 890 & {$[5]$} \\
\hline
\end{tabular}

积变化大, 电极活性物质会发生破裂和粉碎, 导致 电池容量衰减, 循环性能变差。另外, 金属氧化物负 极由于电导率低, 还存在高倍率下充放电容量低等 缺点, 限制了其广泛应用。通常采用两种方法解决 上述问题 ${ }^{[33]}$ : 一种方法是合成纳米结构的金属氧化 物，包括不同的形貌，如纳米粒子、纳米片、纳米线、 纳米棒、纳米管和空心纳米结构。纳米材料可以提 供更短的电子和锂离子传输路径, 从而提高了电导 率和充放电速率。另与块体材料相比, 纳米材料能 够更好的调节由于嵌锂/脱锂造成的机械应变, 从而 提高循环稳定性, 延长循环寿命; 另一种方法就是 制备由金属氧化物/碳纳米材料构成的复合材料电 极。该复合材料电极能够提供足够的空间调节电极 体积在充放电过程中的变化, 并保持电极的机械完 整性, 提高循环的稳定性。Han 等 ${ }^{[00]}$ 发现 CNOs 的 比容量(60 个循环后, 其可逆容量为 $391 \mathrm{mAh} / \mathrm{g}$ )高 于传统石墨材料的比容量, 并把原因归结为三点: 第一, 纳米级的洋苾碳能够提供大的电极-电解液 接触面积, 缩短电子和锂离子的传输路径; 第二, 洋葱碳的多层结构提供了更多的锂存储位; 第三、 洋葱碳周围的非晶碳也对提高比容量有贡献, 因为 无序碳比石墨具有更高的比容量。具有核壳结构的 洋葱碳(核为金属氧化物)是一种良好的负极材料, 主要是因为金属氧化物被包覆后提高了电极材料的 电导率, 并且洋苟碳的石墨壳层能够缓冲充放电过 程中负极体积的变化。表 3 为几种金属氧化物在有 无石墨层包覆情况下的性能比较, 可以看出, 包覆 后的过渡金属氧化物电极的高倍率容量和循环性能 大大提高。

\subsection{OLC 在染料敏化太阳能电池方面的应用}

染料敏化太阳能电池(dye-sensitized solar cell, 即 DSSC)具有制作工艺简单、制作成本低、无污染、光 电转换效率高等优点, 有望替代硅太阳能电池。自 1991, Oregan 制作了第一块染料敏化纳米晶太阳能电 池以来, 20 多年来广大研究者的研究热情从未间断。

DSSC 中对电极有两个作用: 收集从光阳极传 输过来的电子, 并将 $\mathrm{I}_{3}{ }^{-}$还原成 $\mathrm{I}^{-}$。DSSC 对电极材料 必须满足高的导电性、高的比表面积、高的电催化 活性和高化学稳定性等要求, 因此最常用的对电极 材料为铂和碳基材料。铂对电极的转化效率较高, 但其价格昂贵，增加了制造成本。OLC 作为碳基材 料之一, 也是性能良好的对电极材料。图 2 为在同 等测试条件下, 分别以铂和 CNOs 为对电极的相同 结构 DSSC 的光电流-光电压曲线图, 可以看出 CNOs 对电极的性能与 Pt 对电极相当。二者的光电 
表 3 金属氧化物负极材料性能比较

Table 3 Performance comparison of metal oxides used as anode materials

\begin{tabular}{|c|c|c|c|c|}
\hline $\begin{array}{c}\text { Anode } \\
\text { materials }\end{array}$ & $\begin{array}{c}\text { OLC } \\
\text { encapsulation }\end{array}$ & $\begin{array}{l}\text { Initial discharge ability of nanoparticles } \\
\text { electrode at different rates }\end{array}$ & Cycle performance & Ref \\
\hline \multirow{2}{*}{$\mathrm{NiO}$} & No & $\mathrm{NiO}, 2 C, 558.8 \mathrm{mAh} / \mathrm{g}$ & $\begin{array}{c}\text { 0.5C, } 1150 \mathrm{mAh} / \mathrm{g} \text { (initial discharge) and } \\
383.5 \mathrm{mAh} / \mathrm{g} \text { (after } 50 \text { cycles) }\end{array}$ & \multirow{2}{*}{ [3] } \\
\hline & Yes & $\mathrm{NiO} / \mathrm{C}, 2 C, 1105.6 \mathrm{mAh} / \mathrm{g}$ & $\begin{array}{c}0.5 C, 1689.4 \mathrm{mAh} / \mathrm{g} \text { (initial discharge) and } \\
1157.7 \mathrm{mAh} / \mathrm{g} \text { (after } 50 \text { cycles) }\end{array}$ & \\
\hline \multirow[b]{2}{*}{$\mathrm{CuO}$} & No & $\mathrm{CuO}, 1.2 C, 178.9 \mathrm{mAh} / \mathrm{g}$ & $100 \mathrm{~mA} / \mathrm{g}, 270.2 \mathrm{mAh} / \mathrm{g}$ (after 50 cycles) & \multirow[b]{2}{*}[4]{} \\
\hline & Yes & $\mathrm{CuO} / \mathrm{C}, 1.2 C, 535.6 \mathrm{mAh} / \mathrm{g}$ & $\begin{array}{c}100 \mathrm{~mA} / \mathrm{g}, 628.7 \mathrm{mAh} / \mathrm{g} \text { (after } 50 \text { cycles) with a } \\
\text { high Coulombic efficiency of } 98.6 \%\end{array}$ & \\
\hline \multirow{2}{*}{$\mathrm{Co}_{3} \mathrm{O}_{4}$} & No & $\mathrm{Co}_{3} \mathrm{O}_{4}, 2 \mathrm{C}, 459 \mathrm{mAh} / \mathrm{g}$ & $\begin{array}{l}1248.8 \mathrm{mAh} / \mathrm{g} \text { (initial discharge) and } \\
471.5 \mathrm{mAh} / \mathrm{g} \text { (after } 50 \text { cycles) }\end{array}$ & \multirow{2}{*}{ [5] } \\
\hline & Yes & $\mathrm{Co}_{3} \mathrm{O}_{4} / \mathrm{C}, 2 \mathrm{C}, 925 \mathrm{mAh} / \mathrm{g}$ & $\begin{array}{c}\text { 0.5C, } 1467.6 \mathrm{mAh} / \mathrm{g} \text { (initial discharge) and } \\
1026.9 \mathrm{mAh} / \mathrm{g} \text { (after } 50 \text { cycles) }\end{array}$ & \\
\hline \multirow{2}{*}{$\mathrm{SnO}_{2}$} & No & / & $\begin{array}{c}0.2 \mathrm{~mA} / \mathrm{cm}^{2}, 849 \mathrm{mAh} / \mathrm{g} \text { (initial discharge) to } \\
123 \mathrm{mAh} / \mathrm{g} \text { (after } 50 \text { cycles) }\end{array}$ & \multirow{2}{*}{ [63] } \\
\hline & Yes & / & $\begin{array}{c}0.2 \mathrm{~mA} / \mathrm{cm}^{2}, 755 \mathrm{mAh} / \mathrm{g}(\text { initial discharge) and } \\
446 \mathrm{mAh} / \mathrm{g} \text { (after } 50 \text { cycles) }\end{array}$ & \\
\hline
\end{tabular}

转换效率接近, 并且以 CNOs 为对电极的 DSSC 的 填充因子大于 Pt 对电极电池。

\subsection{OLC 在电化学储氢方面的应用}

OLC 具有高的比表面积和电导率, 是碳质储氢 材料的重要成员之一。碳质储氢材料的储氢容量与 比表面积有关，理论储氢容量可以通过公式 mads $\approx$ $S$ spec $\times 2.27 \times 10^{-3} \mathrm{wt} \%$ 估算求得 ${ }^{[64]}$ 。Zhang 等 ${ }^{[20]}$ 在 $850^{\circ} \mathrm{C}$ 下, 以铁镍合金为催化剂, 用 CVD 方法制备 OLC, 再经过 $1100^{\circ} \mathrm{C}$ 高温退火, 制备了中空的 OLC, 然后制作成电极, 在室温下进行电化学储氢性能测 试, 发现 OLC 电极具有优异的电化学活性和放电容 量。在 $500 \mathrm{~mA} / \mathrm{g}$ 的电流密度下, 首次充放电循环达 到最大放电容量约 $590 \mathrm{mAh} / \mathrm{g}$; 经过 10 次充放电循

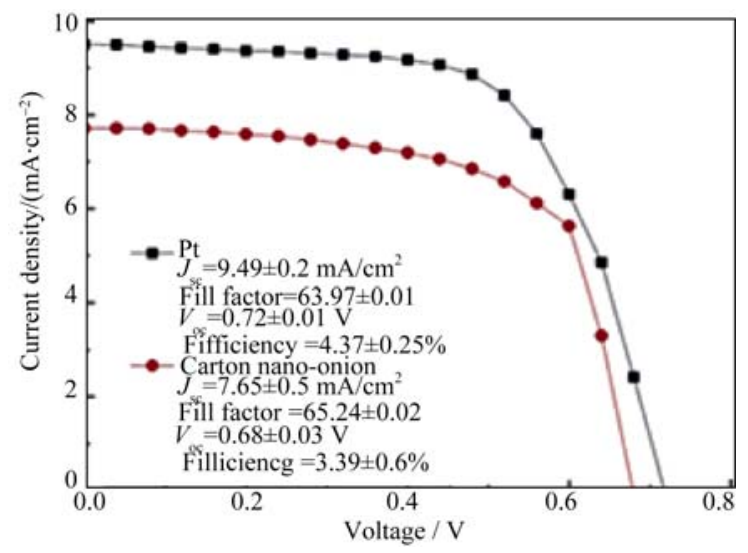

图 2 用 Pt 和 CNOs 做对电极的 DSSCs 的光电流密度-光电 压曲线 ${ }^{[19]}$

Fig. 2 Photocurrent density-photovoltage of the fabricated DSSCs using Pt and CNOs counter electrodes ${ }^{[19]}$
环, 放电容量保持在 $481.6 \mathrm{mAh} / \mathrm{g}$ 以上(对应储氢容 量为 $1.76 \mathrm{wt} \%$ ); 经过 55 次循环，电极仍然保持稳定， 容量保持率仍超过 $80 \%$ 。在高温退火过程中, 金属 颗粒从具有核壳结构的 OLC 中游离出来，导致 OLC 表面形成开口缺陷, 所以中空 OLC 具有较高 放电容量。氢分子可以通过这些开口缺陷扩散到 OLC 的内部中空部分, 大大增加了吸附氢分子的表 面积。Zhang 等 ${ }^{[21]}$ 还利用 CVD 方法制备了三种核壳

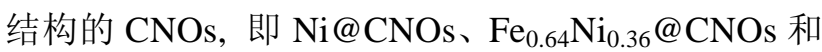
$\mathrm{Fe}_{3} \mathrm{C} @ \mathrm{CNOs}$, 并在室温下进行了电化学储氢性能 测试(如图 3 所示)。结果表明: Ni@CNOs 的储氢容

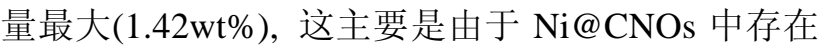
大量的缺陷, 且 $\mathrm{Ni}$ 粒子具有高的电导率和电催化活

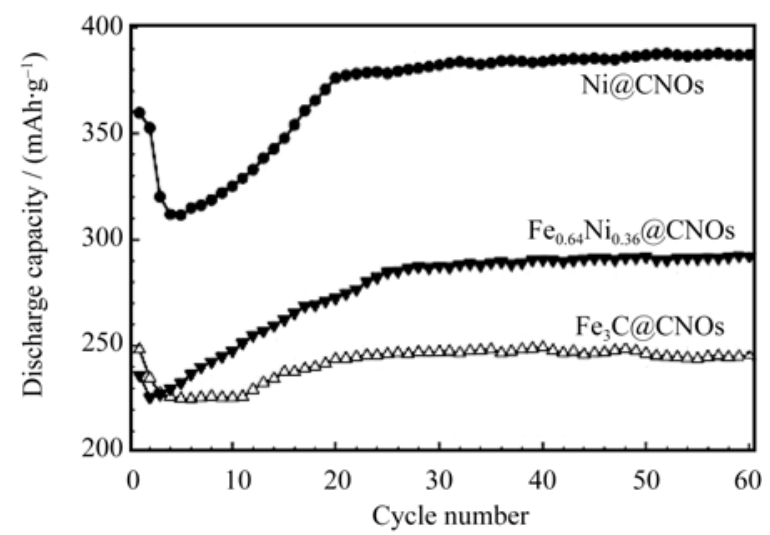

图 3 室温下, 充放电电流密度为 $500 \mathrm{~mA} / \mathrm{g}$ 时三种电极的放 电容量随循环次数的变化 ${ }^{[21]}$

Fig. 3 Discharge capcity of the three types of CNOs versus cycle number at a charge-discharge current density of $500 \mathrm{~mA} / \mathrm{g}$ at room temperature ${ }^{[21]}$ 
性; $\mathrm{Fe}_{3} \mathrm{C} @ \mathrm{CNOs}$ 的储氢容量最小 $\left.(0.91 \mathrm{wt} \%)\right)$, 这主 要是由于 $\mathrm{Fe}_{3} \mathrm{C}$ 电导率低导致 $\mathrm{Fe}_{3} \mathrm{C} @ \mathrm{CNOs}$ 电化学性 能较差。

\subsection{OLC 在超级电容器电极材料方面的应用}

碳材料超级电容器电极的比电容低、能量密度 低, 如何提高碳材料的比电容、能量密度, 是解决问 题的关键。作为碳材料的一员, OLC 电极比电容也 较低, 约 30 40 F/g。提高 OLC 比电容主要有两条 途径。第一条途径是提高 OLC 的比表面积, 可以通 过在制备过程中控制温度 ${ }^{[65]}$ 或后期的化学活化作 用实现。Gao 等 ${ }^{[66]}$ 通过 $\mathrm{KOH}$ 化学活化作用, 得到多 孔 OLC, 提高了比电容和能量密度。同等测试条件 下, 未活化 OLC 电极的比电容、功率密度、能量密 度分别为 $25.8 \mathrm{~F} / \mathrm{g} 、 123 \mathrm{~kW} / \mathrm{kg} 、 1.5 \mathrm{Wh} / \mathrm{kg}$; 活化后 多孔 OLC 电极的比电容、功率密度、能量密度分别 为 $122 \mathrm{~F} / \mathrm{g} 、 153 \mathrm{~kW} / \mathrm{kg} 、 8.5 \mathrm{Wh} / \mathrm{kg}$ 。性能提高主要 是由于化学活化提高了 OLC 的比表面积, 但活化后 的 OLC 在后期的冲洗、超声和过滤过程中, 会损失 大量原材料。第二条途径是制备 OLC 基复合材料电 极, 包括过 OLC/过渡金属氧化物电极和 OLC/导电 聚合物电极等。Anjos 等 ${ }^{[67]}$ 测试了 PQ/OLC 复合材 料电极性能, 发现其能量密度最高可以达到 $4.5 \mathrm{Wh} / \mathrm{kg}$, 大约是 OLC 电极的 9 倍; 比电容最高可 以达到 $264 \mathrm{~F} / \mathrm{g}$, 在恒电流密度 $1.3 \mathrm{~A} / \mathrm{g}$ 下, 经过 10000 次充放电循环, 比电容保持率仍较高。表 3 为不同方法活化的 OLC 电极和 CNOs 复合材料电极 的比电容。

表 3 CNOs 电极和 CNOs 基复合材料电极的比电容

Table 3 Specific capacitances of various CNOs and CNOs-based composite electrode

\begin{tabular}{ccc}
\hline Types of materials & $\begin{array}{c}\text { Specific } \\
\text { capacitances } /\left(\mathrm{F} \cdot \mathrm{g}^{-1}\right)\end{array}$ & Ref \\
\hline $\mathrm{CNOs}$ & 30 & {$[67]$} \\
$\mathrm{CNOs} / \mathrm{KOH}$ activation & $<122$ & {$[66]$} \\
$\mathrm{CNOs} / \mathrm{H}_{2} \mathrm{SO}_{4}$ activation & $20-40$ & {$[68]$} \\
$\mathrm{CNOs} / \mathrm{RuO}_{2} \cdot x \mathrm{H}_{2} \mathrm{O}$ & $96-334$ & {$[69]$} \\
$\mathrm{CNO} / \mathrm{NiO}$ & $218.2-290.6$ & {$[70]$} \\
$\mathrm{CNOs} / \mathrm{Ni}(\mathrm{OH}) 2$ & $727.4-1225.2$ & {$[70]$} \\
$\mathrm{CNOs} / \mathrm{MnO} 2$ & 177.5 & {$[71]$} \\
$\mathrm{CNOs} / \mathrm{PDDA}$ & $20-30$ & {$[72]$} \\
$\mathrm{CNOs} / \mathrm{chit}$ & $20-30$ & {$[72]$} \\
$\mathrm{CNOs} / \mathrm{PANI}$ & $<525$ & {$[73]$} \\
$\mathrm{CNOs} / \mathrm{PQ}$ & 267 & {$[67]$} \\
$\mathrm{CNOs} / \mathrm{NQ}$ & 91 & {$[67]$} \\
$\mathrm{CNOs} / \mathrm{PY}$ & 130 & {$[67]$} \\
\hline
\end{tabular}

\subsection{OLC 在磨擦磨损领域的研究与应用}

碳基润滑剂(石墨、石墨烯、碳纳米管、类金刚石 等)表现出优异的耐磨擦磨损性能, OLC 有望成为性 能更加优异的固体润滑剂, 这是由于 OLC 约 $10 \mathrm{~nm}$ 的 准球形结构, 有可能在纳米尺寸范围内起 “微轴承” 作用; 碳原子排列完美，表面没有悬挂键，OLC 和 偶件材料只可能存在弱的分子间键合; 稳定的封闭 结构使其具有高的机械强度。

Hirata 等 ${ }^{[74]}$ 通过用钢球和硅片做球盘摩擦磨损 试验, 测试了 OLC 的固体润滑性能, 研究表明: (1) 室温下, OLC 在空气和真空中都显示出较稳定的低 摩擦系数 $(<0.1)$, 摩擦系数低于同等测试条件下石 墨、 $\mathrm{C}_{60 / 70}$ 和金刚石团簇的摩擦系数。(2)小尺寸的 OLC 比大尺寸的 OLC 具有更加优异的耐摩擦性能, 这主要是由于大尺寸的 OLC 最外层有更多的缺陷, 会与偶件材料发生键合, 从而造成较大的摩擦系数 和较多的磨损量。

Bucholz 等 ${ }^{[75]}$ 通过原子分子动力学仿真研究了 OLC 的摩擦机理。结果表明, 摩擦性能主要由 OLC 纳米颗粒滚动和滑动所占的比例决定。滚动摩擦变 为滑动摩擦时, 摩擦系数会有一个数量级的增长 (由 0.024 增大到 0.151), 而在摩擦过程中, OLC 滚动 和滑动的相对比例与界面键的形成有关。

\subsection{OLC 在催化方面的应用}

阴极氧还原反应动力学缓慢严重制约了燃料 电池性能的提高。纳米结构的原子掺杂碳材料能够 提高电催化活性和降低成本, 有望取代传统的 $\mathrm{Pt}$ 或 Pt 基电极。常见的掺杂原子有 $\mathrm{N} 、 \mathrm{~S} 、 \mathrm{P}$ 和 $\mathrm{B}$ 等。 比表面积大，且具有多孔结构的共掺杂碳材料在 氧还原反应中具有高的活性。Han 等 ${ }^{[76]}$ 通过一步法 合成了氮、硫共掺杂介孔洋葱碳(NS-MCV), 证实 其在氧还原反应中具有较高的电催化活性, 和商 用 $\mathrm{Pt} / \mathrm{C}$ 催化剂相比, NS-MCV 具有更好的稳定性和 耐受性。

Lin 等 ${ }^{[8]}$ 研究发现氮掺杂的洋芴碳(N-OLC)在苯 乙烯的环氧化反应中显示出良好的催化性能, 其催 化性能优于未掺杂的洋苟碳和一些金属催化剂。

N-OLC 制备方法如图 4 所示, 纳米金刚石在氩气氛 围中经 $1300^{\circ} \mathrm{C}$ 退火 $4 \mathrm{~h}$ 得到洋苟碳; 然后将 OLC 放 入 $120^{\circ} \mathrm{C}$ 的硝酸中, 持续搅拌 $2 \mathrm{~h}$, 所得产物经过过 滤、清洗和烘干, 得到氧掺杂的 OLC(O-OLC); 最后, 在氨气氛中, O-OLC 在不同温度下退火 $4 \mathrm{~h}$ 得到 $\mathrm{N}-\mathrm{OLC}$ 。

OLC 除了在上述领域有着广泛应用外，还可作 为制备纳米栾晶金刚石和聚晶金刚石的前驱物。 


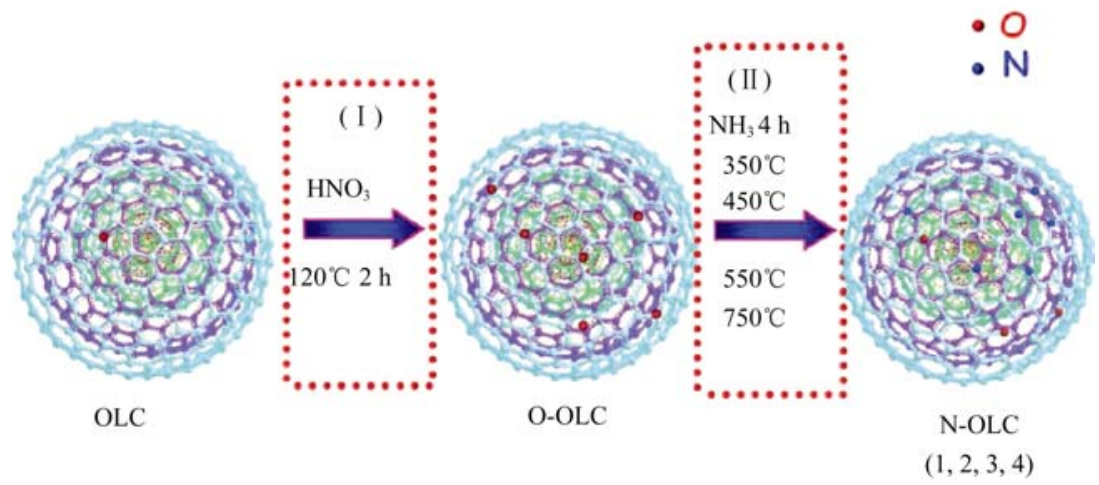

图 4 N-OLC 制备示意图 ${ }^{[24]}$

Fig. 4 Schematic illustration for fabrication N-OLC ${ }^{[24]}$

Huang 等 ${ }^{[77]}$ 以 OLC 为前驱物, 在高温高压下制备出 纳米奕晶金刚石, 其硬度高达 $200 \mathrm{GPa}$, 超过了天 然金刚石。Gong ${ }^{[78]}$ 和 $\mathrm{Yu}^{[79]}$ 以洋葱碳为前驱物, 在 高温高压下制备出了无添加剂的聚晶金刚石, 其硬 度最高可达 55.9 GPa。

\section{3 结束语}

经过多年的研究, 人们已经掌握了多种 OLC 的 制备方法并对其生长机理有了一定的认识, 并且在 应用方面有了一定尝试, 显示出其优异的性能, 但 是仍然存在一些不足:

1) 目前比较成熟的 OLC 的制备方法都存在成 本高、产量低和含有杂质等缺点。快速、简单、低 成本和宏量制备 OLC 以及 OLC 的纯化仍然是以后 的研究重点。

2) OLC 的曲率半径、比表面积、晶化程度与 制备方法、制备条件密切相关，而曲率半径、比表 面积、晶化程度等因素又影响着 OLC 的理化性能, 所以在微观上实现 OLC 的可控生长, 精确控制 OLC 的石墨壳层数、比表面积和晶化程度, 具有重 要意义。

3) OLC 的应用研究才刚刚起步, 仍需要开展大 量的基础研究。优化过渡金属氧化物/OLC 复合材料 电极的制备参数, 进一步提高锂离子二次电池的比 容量、高倍率性能以及循环稳定性能; 研究 OLC 比 表面积、多孔结构、晶化程度对 OLC 对电极性能的 影响, 进一步提高 OLC 对电极的性能; 研究 OLC 比表面积、多孔结构对储氢容量的影响, 通过在 OLC 的表面引入官能团或者掺杂提高氢气和 OLC 的表面结合能, 从而提高储氢容量; 优化 OLC 基复 合材料电极的性能, 提高超级电容器的比电容和能 量密度; 研究不同的元素掺杂以及共掺杂对 OLC 催
化性能的影响等。

OLC 的结构、表面功能化以及掺杂对其性能有 重要影响, 理清它们之间的关系, 优化 OLC 基器件 的性能, 可以推进 OLC 在能源等诸多领域的应用。

\section{参考文献:}

[1] UGARTE D. Curling and closure of graphitic networks under electron-beam irradiation. Nature, 1992, 359: 707-709.

[2] ABDUllaEVA Z, OMURZAK E, IWAMOTO C, et al. Onionlike carbon-encapsulated $\mathrm{Co}, \mathrm{Ni}$, and $\mathrm{Fe}$ magnetic nanoparticles with low cytotoxicity synthesized by a pulsed plasma in a liquid. Carbon, 2012, 50: 1776-1785.

[3] LIU X G, OR S W, JIN C G, et al. NiO/C nanocapsules with onionlike carbon shell as anode material for lithium ion batteries. Carbon, 2013, 60: 215-220.

[4] LIU X G, BI N N, FENG C, et al. Onion-like carbon coated CuO nanocapsules: a highly reversible anode material for lithium ion batteries. Journal of Alloys and Compounds, 2014, 587: 1-5.

[5] LIU X G, OR S W, JIN C G, et al. $\mathrm{Co}_{3} \mathrm{O}_{4} / \mathrm{C}$ nanocapsules with onionlike carbon shells as anode material for lithium ion batteries. Electrochimica Acta, 2013, 100: 140-146.

[6] QIAO Z J, LI J J, ZHAO N Q, et al. Graphitization and microstructure transformation of nanodiamond to onion-like carbon. Scripta Materialia, 2006, 54: 225-229.

[7] XU B S. Prospects and research progress in nano onion-like fullerenes. New carbon materials, 2008, 23(4): 289-301.

[8] LIN Y M, PAN X L, QI W, et al. Nitrogen-doped onion-like carbon: a novel and efficient metal-free catalyst for epoxidation reaction. J. Mater. Chem. A, 2014, 2: 12475-12483.

[9] BOGDANOV K, FEDOROV A, OSIPOV V, et al. Annealinginduced structural changes of carbon onions: high-resolution transmission electron microscopy and Raman studies. Carbon, 2014, 73: 78-86.

[10] XU B S, TANAKA S I. Multiple-nuclei Onion Like Fullerenes 
Cultivated by Electron Beam Irradiation. Proc. Int. Conf. ICSE, Cambridge, 1997: 355-360.

[11] WANG Q, SUN X L, HE D Y, et al. Preparation and study of carbon nano-onion for lithium storage. Materials Chemistry and Physics, 2013, 139: 333-337.

[12] WANG Y, HAN Z J, YU S F, et al. Core-leaf onion-like carbon/ $\mathrm{MnO}_{2}$ hybrid nano-urchins for rechargeable lithium-ion batteries. Carbon, 2013, 64: 230-236.

[13] WU G, NELSON M, MA S G, et al. Synthesis of nitrogen-doped onion-like carbon and its use in carbon-based CoFe binary non-precious-metal catalysts for oxygen-reduction. Carbon, 2011, 49: 3972-3982.

[14] MATSUMOTO N, JOLY-POTTUZ L, KiNOSHITA H, et al. Application of onion-like carbon to micro and nanotribology. Diamond \& Related Materials, 2007, 16: 1227-1230.

[15] JOLY-POTTUZ L, MATSUMOTO N, KINOSHITA H, et al. Diamond-derived carbon onions as lubricant additives. Tribology International, 2008, 41: 69-78.

[16] MATSUMOTO N, MISTRY K K, KIM J H, et al. Friction reducing properties of onion-like carbon based lubricant under high contact pressure. Tribology, 2012, 6(3): 116-120.

[17] WANG Y, XING G Z, HAN Z J, et al. Pre-lithiation of onion-like carbon/MoS 2 nano-urchin anodes for high-performance rechargeable lithium ion batteries. Nanoscale, 2014, 6: 8884-8890.

[18] WANG Y, YAN F, LIU S W, et al. Onion-like carbon matrix supported $\mathrm{Co}_{3} \mathrm{O}_{4}$ nanocomposites: a highly reversible anode material for lithium ion batteries with excellent cycling stability. $J$. Mater. Chem. A, 2013, 1(17): 5212-5216.

[19] BU I Y Y. Synthesis of graphitic carbon nano-onions for dye sensitized solar cells. Solar Energy, 2014, 105: 236-242.

[20] ZHANG C G, LI J J, LIU E Z, et al. Synthesis of hollow carbon nano-onions and their use for electrochemical hydrogen storage. Carbon, 2012, 50: 3513-3521.

[21] ZHANG C G, LI J J, SHI C S, et al. Effect of Ni, Fe and Fe-Ni alloy catalysts on the synthesis of metal contained carbon nano-onions and studies of their electrochemical hydrogen storage properties. Journal of Energy Chemistry, 2014, 23: 324-330.

[22] MCDONOUGH J K, FROLOV A I, PRESSER V, et al. Influence of the structure of carbon onions on their electrochemical performance in supercapacitor electrodes. Carbon, 2012, 50: 3298-3309.

[23] PECH D, BRUNET M, DUROU H, et al. Ultrahigh-power micrometre-sized supercapacitors based on onion-like carbon. $\mathrm{Na}$ ture Nanotechnology, 2010, 5: 651-654.

[24] BUSHUEVA E G, GALKIN P S, BULUSHEVA L G, et al. Double layer supercapacitor properties of onion-like carbon materials. Phys. Stat. Sol. (b), 2008, 245(10): 2296-2299.
[25] SHENDEROVA O, GRISHKO V, CUNNINGHAM G, et al. Onionlike carbon for terahertz electromagnetic shielding. Diamond \& Related Materials, 2008, 17: 462-466.

[26] SANO N, WANG H, ALEXANDROU I, et al. Properties of carbon onions produced by an arc discharge in water. Journal of Applied Physics, 2002, 92(5): 2783-2788.

[27] BORGOHAIN R, YANG J, SELEGUE J P, et al. Controlled synthesis, efficient purification, and electrochemical characterization of arc-discharge carbon nano-onions. Carbon, 2014, 66: $272-284$.

[28] XING G, JIA X L, SHI Z Q. The production of carbon nano-materials by arc discharge under water or liquid nitrogen . New Carbon Materials, 2007, 22(4): 337-341.

[29] SANO N, WANG H, CHHOWALLA M, et al. Nanotechnology: synthesis of carbon 'onions' in water. Nature, 2001, 414: 506-507.

[30] ZHOU J F, SHEN Z Y, HOU S M, et al. Adsorption and manipulation of carbon onions on highly oriented pyrolytic graphite studied with atomic force microscopy. Applied Surface Science, 2007, 253: 3237-3241.

[31] LIU W, MIAO Y, MENG Q S. Synthesis of Onion-Like Fullerenes by Arc Discharge in Non-Toxic Organic Liquid. Integrated Ferroelectrics, 2012, 138: 77-82.

[32] CHEN X H, DENG F M, WANG J X, et al. New method of carbon onion growth by radio-frequency plasma-enhanced chemical vapor deposition. Chemical Physics Letters, 2001, 336: 201-204.

[33] SZERENCSI M, RADNOCZI G. The mechanism of growth and decay of carbon nano-onions formed by ordering of amorphous particles. Vacuum, 2010, 84: 197-201.

[34] HE C N, TIAN F, LIU S J, et al. Characterization and magnetic property of carbon coated metal nanoparticles and hollow carbon onions fabricated by CVD of methane. Materials Letters, 2008, 62: 3697-3699.

[35] HE C N, ZHAO N Q, DU X W, et al. Low-temperature synthesis of carbon onions by chemical vapor deposition using a nickel catalyst supported on aluminum. Scripta Materialia, 2006, 54: 689-693.

[36] WANG X M, XU B S, LIU X G, et al. Synthesis of Fe-included onion-like fullerenes by chemical vapor deposition. Diamond \& Related Materials, 2006, 15: 147-150.

[37] ZHANG C G, LI J J, SHI C S, et al. The efficient synthesis of carbon nano-onions using chemical vapor deposition on an unsupported Ni-Fe alloy catalyst. Carbon, 2011, 49: 1151-1158.

[38] MYKHAYLYK O O, SOLONIN Y M, BATCHELDER D N, et al. Transformation of nanodiamond into carbon onions: a comparative study by high-resolution transmission electron microscopy, electron energy-loss spectroscopy, X-ray diffraction, small-angle 
X-ray scattering, and ultraviolet Raman spectroscopy. Journal of applied Physics, 2005, 97: 074302-1-6.

[39] BUTENKO Y V, KRISHNAMURTHY S, CHAKRABORTY A K, et al. Photoemission study of onionlike carbons produced by annealing nanodiamonds. Physical Review B, 2005, 71: 075420-1-10.

[40] CEBIK J, MCDONOUGH J K, PEERALLY F, et al. Raman spectroscopy study of the nanodiamond-to-carbon onion transformation. Nanotechnology, 2013, 24: 205703-1-10.

[41] BYSTRZEJEWSKI M, RUMMELI M H, GEMMING T, et al. Catalyst-free synthesis of onion-like carbon nanoparticles. New Carbon Materials, 2010, 25(1): 1-8.

[42] GORELIK T, URBAN S, FALK F, et al. Carbon onions produced by laser irradiation of amorphous silicon carbide. Chemical Physics Letters, 2003, 373: 642-645.

[43] HU S L, BAI P K, TIAN F, et al. Hydrophilic carbon onions synthesized by millisecond pulsed laser irradiation. Carbon, 2009, 47: 876-883.

[44] DOROBAnTU D, BOTA P M, BOERASU I, et al. Pulse laser ablation system for carbon nano-onions fabrication. Surface Engineering and Applied Electrochemistry, 2014, 50(5): 390-394.

[45] ZHANG H M, LIANG C H, LIU J, et al. The formation of onion-like carbon-encapsulated cobalt carbide core/shell nanoparticles by the laser ablation of metallic cobalt in acetone. Carbon, 2013, 55: 108-115.

[46] GAO Y, ZHOU Y S, PARK J B, et al. Resonant excitation of precursor molecules in improving the particle crystallinity, growth rate and optical limiting performance of carbon nanoonions. Nanotechnology, 2011, 22(16): 165604-1-6.

[47] XIAO J, OUYANG G, LIU P, et al. Reversible nanodiamondcarbon onion phase transformations. Nano letters, 2014, 14: 3645-3652.

[48] CABIOC'H T, JAOUEN M, THUNE E, et al. Carbon onions formation by high-dose carbon ion implantation into copper and silver. Surface and Coatings Technology, 2000, 128-129: 43-50.

[49] HUANG J Y, YASUDA H AND MORI H. Highly curved carbon nanostructures produced by ball-milling. Chemical Physics Letters, 1999, 303: 130-134.

[50] HAN F D, YAO B AND BAI Y J. Preparation of carbon nano-onions and their application as anode materials for rechargeable lithium-ion batteries. J. Phys. Chem. C, 2011, 115: 8923-8927.

[51] GHOSH M, SONKAR S K, SAXENA M, et al. Carbon nano-onions for imaging the life cycle of drosophila melanogaster. Small, 2011, 7(22): 3170-3177.

[52] CHOUCAIR M, STRIDE J A. The gram-scale synthesis of carbon onions. Carbon, 2012, 50: 1109-1115.
[53] HE C N, WU S, ZHAO N Q, et al. Carbon-encapsulated $\mathrm{Fe}_{3} \mathrm{O}_{4}$ nanoparticles as a high-rate lithium ion battery anode material. ACS Nano, 2013, 7(5): 4459-4469.

[54] HAN Q Y, ZAI J T, XIAO Y L, et al. Direct growth of $\mathrm{SnO}_{2}$ nanorods on graphene as high capacity anode materials for lithium ion batteries. RSC Adv., 2013, 3: 20573-20578.

[55] FENG J K, XIONG S L, QIAN Y T, et al. Synthesis of nanosized cadmium oxide $(\mathrm{CdO})$ as a novel high capacity anode material for Lithium-ion batteries: influence of carbon nanotubes decoration and binder choice. Electrochimica Acta, 2014, 129: 107-112.

[56] GUO B K, CHI M F, SUN X G, et al. Mesoporous carbon- $\mathrm{Cr}_{2} \mathrm{O}_{3}$ composite as an anode material for lithium ion batteries. Journal of Power Sources, 2012, 205: 495-499.

[57] CHEN A, LI C X, TANG R, et al. $\mathrm{MoO}_{2}$-ordered mesoporous carbon hybrids as anode materials with highly improved rate capability and reversible capacity for lithium-ion battery. Phys. Chem. Chem. Phys., 2013, 15: 13601-13610.

[58] LIU B, HU X L, XU $\mathrm{H} \mathrm{H}$, et al. Encapsulation of $\mathrm{MnO}$ nanocrystals in electrospun carbon nanofibers as high-performance anode materials for lithium-ion batteries. Scientific Reports, 2014, 4: 4229.

[59] ZANG J, CHEN J J, ZHANG C L, et al. The synthesis of a core-shell $\mathrm{MnO}_{2} / 3 \mathrm{D}$-ordered hollow carbon sphere composite and its superior electrochemical capability for lithium ion batteries. J. Mater. Chem. A, 2014, 2(18): 6343-6347.

[60] LEPPLE M, ADAM R, CUPID D M, et al. Thermodynamic investigations of copper oxides used as conversion type electrodes in lithium ion batteries. J. Mater. Sci., 2013, 48: 5818-5826.

[61] WANG J, ZHAO $\mathrm{H}$ L, ZENG Z P, et al. Nano-sized $\mathrm{Fe}_{3} \mathrm{O}_{4}$ /carbon as anode material for lithium ion battery. Materials Chemistry and Physics, 2014, 148: 699-704.

[62] QIN D, YAN P, LI G Z, et al. Synthesis of hierarchical CoO nano/microstructures as anode materials for lithium-ion batteries. Journal of Nanomaterials, 2014, 2014: 489862-1-5.

[63] ZHANG H J, SONG H H, ZHOU J S, et al. Preparation and electrochemical properties of $\mathrm{SnO}_{2}$ /onion-like hollow carbon nanoparticle composites as anode materials for lithium-ion batteries. Acta Phys.-Chim. Sin., 2010, 26(5): 1259-1263.

[64] STROBEL R, GARCHE J, MOSELEY P T, et al. Hydrogen storage by carbon materials. Journal of Power Sources, 2006, 159: 781-801.

[65] PORTET C, YUSHIN G, GOGOTSI Y. Electrochemical performance of carbon onions, nanodiamonds, carbon black and multiwalled nanotubes in electrical double layer capacitors. Carbon, 2007, 45: 2511-2518.

[66] GAO Y, ZHOU Y S, QIAN M, et al. Chemical activation of car- 
bon nano-onions for high-rate supercapacitor electrodes. Carbon, 2013, 51: 52-58.

[67] ANJOS D M, MCDONOUGH J K, PERRE E, et al. Pseudocapacitance and performance stability of quinone-coated carbon onions. Nano energy, 2013, 2: 702-712.

[68] PLONSKA-BRZEZINSKA M E, ECHEGOYEN L. Carbon nano-onions for supercapacitor electrodes: recent developments and applications. Journal of Materials Chemistry A, 2013, 1: 13703-13714.

[69] BORGOHAIN R, LI J C, SELEGUE J P, et al. Electrochemical study of functionalized carbon nano-onions for high-performance supercapacitor electrodes. J. Phys. Chem. C, 2012, 116: 15068-15075.

[70] PLONSKA-BRZEZINSKA M E, BRUS D M, MOLINAONTORIA A, et al. Synthesis of carbon nano-onion and nickel hydroxide/oxide composites as supercapacitor electrodes. RSC Adv., 2013, 3: 25891-25901.

[71] WANG Y, YU S F, SUN C Y, et al. $\mathrm{MnO}_{2} /$ onion-like carbon nanocomposites for pseudocapacitors. J. Mater. Chem., 2012, 22: 17584-17588.

[72] BRECZKO J, WINKLER K, PLONSKA-BRZEZINSKA M E, et al. Electrochemical properties of composites containing small carbon nano-onions and solid polyelectrolytes. J. Mater. Chem.,
2010, 20: 7761-7768.

[73] PLONSKA-BRZEZINSKA D M E, BRECZKO J, PALYS D B, et al. The electrochemical properties of nanocomposite films obtained by chemical in situ polymerization of aniline and carbon nanostructures. ChemPhysChem, 2013, 14(1): 116-124.

[74] HIRATA A, IGARASHI M, KAITO T. Study on solid lubricant properties of carbon onions produced by heat treatment of diamond clusters or particles. Tribology International, 2004, 37: 899-905.

[75] BUCHOLZ E W, PHILlPOT S R, SINNOTT S B. Molecular dynamics investigation of the lubrication mechanism of carbon nano-onions. Computational Materials Science, 2012, 54: 91-96.

[76] HAN C, BO X J, ZHANG Y F, et al. One-pot synthesis of nitrogen and sulfur co-doped onion-like mesoporous carbon vesicle as an efficient metal-free catalyst for oxygen reduction reaction in alkaline solution. Journal of Power Sources, 2014, 272: 267-276.

[77] HUANG Q, YU D L, XU B, et al. Nanotwinned diamond with unprecedented hardness and stability. Nature, 2014, 510: 250-253.

[78] 龚 文. 碳纳米葱无添加剂高温高压烧结聚晶金刚石的研究. 秦皇岛: 燕山大学硕士学位论文, 2012.

[79] 余强华. 微米金刚石对碳纳米䓤烧结 PCD 组织及性能的影响. 秦皇岛: 燕山大学硕士学位论文, 2013. 